\title{
Clinical validation of a 90-gene expression test for tumor tissue of origin diagnosis: a large-scale multicenter study of 1417 patients
}

Wei Sun ${ }^{1+}$, Wei Wu ${ }^{2+}$, Qifeng Wang ${ }^{3,4,5,6+}$, Qian Yao' ${ }^{1}$ Qin Feng ${ }^{1}$, Yue Wang ${ }^{1}$, Yu Sun ${ }^{1}$, Yunying Liu², Qian Lai ${ }^{2}$, Gu Zhang ${ }^{2}$, Peng Qi ${ }^{3,4,5,6}$, Yifeng Sun ${ }^{7}$, Chenhui Qian ${ }^{7}$, Wanli Ren ${ }^{7}$, Zhengzhi Luo ${ }^{7}$, Jinying Chen ${ }^{7}$, Hongying Wang ${ }^{7}$, Qinghua Xu $\mathrm{u}^{6,7,8,9} \mathbb{D}$, Xiaoyan Zhou ${ }^{3,4,5,6^{*}}$, Wenyong Sun ${ }^{2^{*}}$ and Dongmei Lin ${ }^{1^{*}}$

\begin{abstract}
Background: Once malignancy tumors were diagnosed, the determination of tissue origin and tumor type is critical for clinical management. Although the significant advance in imaging techniques and histopathological approaches, the diagnosis remains challenging in patients with metastatic and poorly differentiated or undifferentiated tumors. Gene expression profiling has been demonstrated the ability to classify multiple tumor types. The present study aims to assess the performance of a 90-gene expression test for tumor classification (i.e. the determination of tumor tissue of origin) in real clinical settings.
\end{abstract}

Methods: Formalin-fixed paraffin-embedded samples and associated clinicopathologic information were collected from three cancer centers between January 2016 and January 2021. A total of 1417 specimens that met quality control criteria (RNA quality, tumor cell content $\geq 60 \%$ and so on) were analyzed by the 90 -gene expression test to identify the tumor tissue of origin. The performance was evaluated by comparing the test results with histopathological diagnosis.

Results: The 1417 samples represent 21 main tumor types classified by common tissue origins and anatomic sites. Overall, the 90 -gene expression test reached an accuracy of $94.4 \%$ (1338/1417, 95\% Cl: 0.93 to 0.96). Among different tumor types, sensitivities were ranged from 74.2\% (head\&neck tumor) to 100\% (adrenal carcinoma, mesothelioma, and prostate cancer). Sensitivities for the most prevalent cancers of lung, breast, colorectum, and gastroesophagus are 95.0\%, 98.4\%, 93.9\%, and 90.6\%, respectively. Moreover, specificities for all 21 tumor types are greater than 99\%.

\footnotetext{
*Correspondence: 13524324387@163.com; sunwy@zjcc.org.cn; lindm3@163.com

tWei Sun, Wei Wu and Qifeng Wang contributed equally to this work ${ }^{1}$ Department of Pathology, Key Laboratory of Carcinogenesis and Translational Research (Ministry of Education), Peking University Cancer Hospital \& Institute, No.52 Fucheng Road, Wu Ke Song, Haidian District, Beijing, China

${ }^{2}$ Department of Pathology, Cancer Hospital of the University of Chinese

Academy of Sciences (Zhejiang Cancer Hospital), No.1 East Road of Banshan, Hangzhou, Zhejiang, China

${ }^{3}$ Department of Pathology, Fudan University Shanghai Cancer Center, No.270 Dong'An Road, Shanghai, China

Full list of author information is available at the end of the article
}

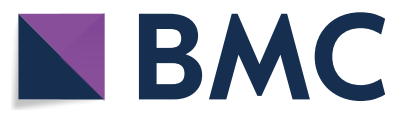

(1) 2022. Open Access This article is licensed under a Creative Commons Attribution 4.0 International License, which permits use, sharing, adaptation, distribution and reproduction in any medium or format, as long as you give appropriate credit to the original author(s) and the source, provide a link to the Creative Commons licence, and indicate if changes were made. The images or other third party material in this article are included in the article's Creative Commons licence, unless indicated otherwise in a credit line to the material. If material is not included in the article's Creative Commons licence and your intended use is not permitted by statutory regulation or exceeds the permitted use, you will need to obtain permission directly from the copyright holder. To view a copy of this licence, visit http://creativecommons.org/licenses/by/4.0/. The Creative Commons Public Domain Dedication waiver (http://creativecommons.org/publicdomain/zero/1.0/) applies to the data made available in this article, unless otherwise stated in a credit line to the data. 
Conclusions: These findings showed robust performance of the 90-gene expression test for identifying the tumor tissue of origin and support the use of molecular testing as an adjunct to tumor classification, especially to those poorly differentiated or undifferentiated tumors in clinical practice.

Keywords: Tissue of origin, Gene expression profiling, Real-time PCR, Tumor classification, The 90-gene expression assay

\section{Introduction}

The cancer burden is rising rapidly due to the aging of the population and the adoption of unhealthy lifestyle behaviors, which became the leading cause of death in China [1]. Once malignancy tumors were diagnosed, the determination of tissue origin and tumor type is critical for clinical management. In routine clinical practice, tumor diagnosis requires a comprehensive synthesis of the clinical and pathological findings. At present, although the significant advance in imaging techniques and histopathological approaches, including morphology and immunohistochemistry (IHC), the diagnosis remains challenging in patients, which initially presenting with metastatic and poorly differentiated or undifferentiated tumors [2-5].

In the past decade, different approaches based on gene expression profiling, DNA methylation, and genomic alteration were developed to identify tumor tissue of origin [6-8]. Many of these assays compared the molecular profiles of the test sample as determined by either microarray, next-generation sequencing (NGS), or real-time PCR (RT-PCR) to molecular profiles of tumors with confirmed tumor types. Two commercialized assays termed Tissue of Origin (TOO) (Vyant Bio, New Jersey, USA) and CancerTYPE ID (Biotheranostics, San Diego, CA, USA) were commonly performed after the failure of the morphological and IHC assessment [9, 10]. The clinical utility of these two assays has been evaluated in few validation studies with an overall sensitivity of $87 \%$ to $87.8 \%$, which is favorable to the histopathological method [9, 10].

In our previous study, a 90-gene expression assay was developed to identify 21 common tumor types using RTPCR methods with total RNA isolated from formalinfixed, paraffin-embedded (FFPE) tumor tissue [7]. The tumors originated from 21 tissue types, including adrenal gland, brain, breast, cervix, colorectum, endometrium, gastroesophagus, germ cell, head\&neck, kidney, liver, lung, melanoma, mesothelioma, neuroendocrine, ovary, pancreas, prostate, sarcoma, thyroid, and urinary system. In a retrospective cohort of 609 clinical specimens, the 90-gene expression assay demonstrated an overall agreement of $90.4 \%$ for primary tumors and $89.2 \%$ for metastatic tumors. Several studies also demonstrated the excellent performance of the 90-gene expression assay in differentiation diagnosis of triple-negative breast cancer, metastatic brain tumor, squamous cell carcinoma, multiple primary tumors, etc. [11-14]. In the present study, we conducted a large-scale, multicenter study to evaluate the performance of the 90-gene expression assay for tumor tissue of origin identification in real clinical settings.

\section{Materials and methods}

\section{Ethics statement}

The study was conducted under protocols approved by the institutional review boards of each institution, including Beijing Cancer Hospital (BCH, Beijing, China), Fudan University Shanghai Cancer Center (FUSCC, Shanghai, China), and Cancer Hospital of the University of Chinese Academy of Sciences, Zhejiang Cancer Hospital (ZCH, Hangzhou, China). All patients signed informed consent.

\section{Case selection}

In this study, we enrolled a total of 1540 patients between January 2016 and January 2021 from three institutions in China. The inclusion criteria for the multisite study were the following: (1) surgical specimen including primary or metastatic tumors; (2) histologically confirmed tumor type; (3) diagnosis contained within the 21 main tumor types; (4) FFPE tumor specimens processed less than three years from the time of testing; (5) at least $60 \%$ tumor cell content available on the hematoxylin and eosin (H\&E) stained slide; (6) less than $40 \%$ necrosis. Exclusion criteria were (1) tumor specimens obtained after chemotherapy or radiotherapy; (2) cytology cases, biopsy (needle core biopsy [NCB] or fine-needle aspiration [FNA]) cases and decalcified cases. All samples were deidentified, assigned internal accession numbers. The technicians performed the 90-gene expression assay in each institution. Investigators who interpreted the test results were blinded to patients' medical history, sample location, and histopathological information.

\section{RNA extraction}

For cases meeting the inclusion and exclusion criteria, 5 to $155 \mu \mathrm{m}$ unstained sections were freshly cut for total RNA isolation. The regions of tumor tissue were marked on the H\&E-stained slides by senior pathologists at each center (W S and Q Y in BCH, QF W in FUSCC, W W and $\mathrm{YY} \mathrm{L}$ in $\mathrm{ZCH}$ ). Tumor cells were then enriched by 
macro-dissected manually. Total RNA was isolated using FFPE Total RNA Isolation Kit (Canhelp Genomics Co., Ltd, Hangzhou, China) as described before [7]. The concentration and purity of total RNA were measured by spectrophotometer. Exclusion criteria were insufficient RNA (concentration of total RNA, $<60 \mathrm{ng} / \mu \mathrm{l}$ ) and low purity (A260/A280 ratio, $>2.1$ or $<1.7$ ).

\section{Gene expression profiling and classification algorithm}

The 90-gene expression assay (Canhelp Genomics Co., Ltd) was carried out as previously described [7]. In brief, the reverse transcription was performed on isolated total RNA. Next, the RT-PCR reaction was applied with a 7500 Real Time PCR System (Applied Biosystems) to perform tumor-specific gene expression profiling. The internal control (IC) gene was used to assess the sample quality, while a weak RT-PCR signal (cycle threshold [Ct] value of the IC, greater than 38) was excluded. Additionally, no template control (NTC) was used to evaluate the potential PCR reaction contamination. The sample was excluded when the Ct of the NTC was less than 38 .

For each case, the 90-gene classifier analyzed the gene expression pattern of the 90 tumor-specific genes and generated similarity scores for each primary tumor type based on the degree of similarities of the test specimen to the gene expression database. The range of similarity scores was 0 (low similarity) to 100 (high similarity) for each tumor type, and the sum of similarity scores across 21 tumor types was 100 .

\section{Statistical analysis}

The internal accession numbers of all cases were finally broken, and test results predicted by the 90-gene expression assay were compared with the reference diagnosis to evaluate the assay performance. As for each tumor type in the panel, sensitivity (or positive percent agreement) was defined as the ratio of true positive results to the total positive samples analyzed. Specificity (or negative percent agreement) was defined as the ratio of true negative results to the total negative samples analyzed. A confusion matrix was generated for each tumor type. All statistical analyses were computed in R software (version 3.6.1). All statistical tests were two-sided, and values of p-value less than 0.05 were considered statistically significant.

\section{Results}

\section{Patients and specimens}

As shown in Fig. 1, 1540 specimens were enrolled from three cancer centers. Among these cases, 23 cases were excluded due to non-sufficient RNA for analysis and/or lower purity, 92 cases had severely degraded nucleic acid, and 8 cases were ruled out due to potential reaction contamination. A total of 1417 samples met all criteria and entered into the study with an overall analytical success rate of $92.0 \%$ (1417 of 1540). For details, 924 samples were processed retrospectively during October 2018 and March 2021 (retrospective cohort). In addition, 493 samples with mainly poorly differentiated and undifferentiated tumors were prospectively analyzed from October 2020 to January 2021 in a consecutive manner (prospective cohort). The patients' characteristics according to main tumor types are summarized in Table 1 . The median age of the entire patient was 57 years old, ranging from 9 to 88 . There were $673(47.5 \%)$ males and 744 (52.5\%) females with a sex ratio of 1:1.1. There were 1226 primary tumors and 191 metastatic tumors. As for histological type, the most common type was adenocarcinoma $(\mathrm{N}=943,66.5 \%)$, followed by squamous cell carcinoma $(\mathrm{N}=166,11.7 \%)$, urothelial carcinoma $(\mathrm{N}=55,3.9 \%)$, melanoma $(\mathrm{N}=54,3.8 \%)$, neuroendocrine tumor $(\mathrm{N}=52,3.7 \%)$, tumor $(\mathrm{N}=49,3.5 \%)$, sarcoma $(\mathrm{N}=46$, $3.2 \%)$, germ cell tumor $(\mathrm{N}=40,2.8 \%)$ and mesothelioma $(\mathrm{N}=12,0.9 \%)$. Of 1417 specimens, the histologic grades of 1112 were assigned, $37.6 \%(\mathrm{~N}=418)$ were well-moderately differentiated, and $62.4 \%(\mathrm{~N}=694)$ were poorly differentiated or undifferentiated. The distribution of tumor types in the entire cohort and three institutions were shown in Fig. 2. The most common primary sites included the lung $(\mathrm{N}=141,10.0 \%)$, breast $(\mathrm{N}=123$, $8.7 \%)$, colorectum $(\mathrm{N}=114,8.0 \%)$, and gastroesophagus $(\mathrm{N}=106,7.5 \%)$.

\section{Overall accuracy of the 90-gene expression assay for tumor classification}

The 90-gene expression assay results showed an overall agreement of $94.4 \%$ (1338/1417, 95\% CI: 0.93 to 0.96 ) compared with the pathological diagnosis. The performance of the 90-gene expression assay for each tumor type was shown in Table 2. Of the different tumor types, the sensitivities were ranged from $74.2 \%$ (head\&neck) to 100\% (adrenal, mesothelioma, and prostate). Sensitivities for the most prevalent cancers of lung, breast, colorectum, and gastroesophagus are $95.0 \%, 98.4 \%, 93.9 \%$, and $90.6 \%$, respectively. Overall, 18 out of 21 tumor types had sensitivities greater than $90 \%$, and all 21 tumor types had specificities greater than $99 \%$. A confusion matrix of the relationship of predicted results and reference diagnosis was shown in Fig. 3.

\section{Analysis by clinicopathological subsets}

The performance of the 90-gene expression assay in clinicopathological subsets was shown in Table 3. In the present study, retrospective cohort $(\mathrm{N}=924)$ and 


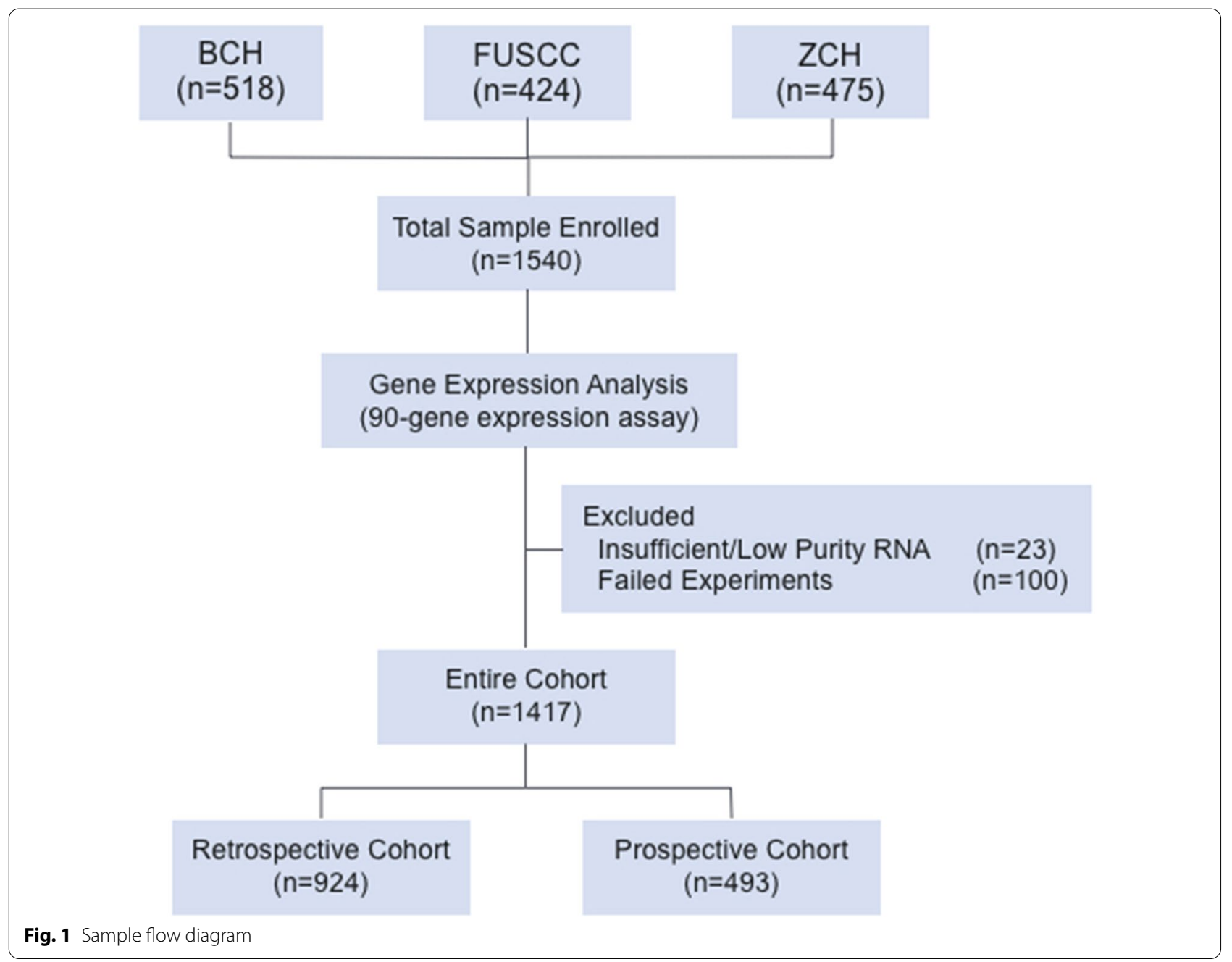

prospective cohort $(\mathrm{N}=493)$ were established to comprehensively evaluate the performance of the 90-gene expression assay in real clinical settings. Accuracy from the prospective cohort was slightly lower than the retrospective cohort $(92.1 \%$ versus $95.7 \%$, p-value $=0.007)$. In an analysis comparing well-moderately differentiated and poorly differentiated or undifferentiated tumors, the 90-gene expression assay showed satisfying performance for accurate identification of a primary site, 95.5\% (399 of 418) for well-moderately differentiated tumors and $94.5 \%$ (656 of 694) for poorly differentiated or undifferentiated tumors, with no statistically different $(\mathrm{p}$-value $=0.59$ ). Among different histological types, agreement rates between the 90-gene expression assay predictions and the reference diagnosis were $95.2 \%$ (898 of 943) for adenocarcinoma, $91.0 \%$ (151 of 166) for squamous cell carcinoma, 95.4\% (53 of 55) for urothelial carcinoma, 88.9\% (48 of 54) for melanoma, $94.2 \%$ (49 of 52) for neuroendocrine tumor and $91.8 \%$ (45 of 49 ) for tumor, $93.5 \%$ (43 of 46 ) for sarcoma, 97.5\% (39 of 40) for germ cell tumor and $100 \%$ (12 of 12 ) for mesothelioma ( $\mathrm{p}$-value $=0.23$ ). For the squamous cell carcinomas $(\mathrm{N}=166)$ originated from cervix $(\mathrm{N}=89)$, head\&neck $(\mathrm{N}=31)$, gastroesophageal $(\mathrm{N}=27)$, and lung $(\mathrm{N}=19)$, the agreements for tumor classification were $98.9 \%$ (88 of 89 ), $74.2 \%$ (23 of 31 ), $85.2 \%$ (23 of 27) and 89.5\% (17 of 19), respectively. Of 52 neuroendocrine tumor cases, their tissue of origins was composed of the thyroid $(\mathrm{N}=23)$, pancreas $(\mathrm{N}=10)$, lung $(\mathrm{N}=7)$, cervix $(2)$, skin $(\mathrm{N}=1)$, urinary $(\mathrm{N}=1)$, colorectum $(\mathrm{N}=1)$, and undefined $(\mathrm{N}=7)$. The overall accuracy for neuroendocrine tumors reached $94.2 \%$. In addition, the overall accuracy of the three study sites was 94.4\% (489 of 518) at BCH, $95.0 \%$ (403 of 424) at FUSCC, and $93.9 \%$ (446 of 475 ) at $\mathrm{ZCH}$. The assay performance across different centers was not statistically different $(\mathrm{p}$-value $=0.75)$. 
Table 1 Patient and tumor demographics and specimen sources

\begin{tabular}{|c|c|c|}
\hline Characteristics & $\begin{array}{l}\text { Number of } \\
\text { specimens } \\
(\mathrm{N}=1417)\end{array}$ & Percentage (\%) \\
\hline \multicolumn{3}{|l|}{ Study } \\
\hline Retrospective & 924 & 65.2 \\
\hline Prospective & 493 & 34.8 \\
\hline \multicolumn{3}{|l|}{ Centers } \\
\hline $\mathrm{BCH}$ & 518 & 36.6 \\
\hline FUSCC & 424 & 29.9 \\
\hline $\mathrm{ZCH}$ & 475 & 33.5 \\
\hline \multicolumn{3}{|l|}{ Age (year) } \\
\hline Median & 57 & \\
\hline Range & $9-88$ & \\
\hline \multicolumn{3}{|l|}{ Gender } \\
\hline Male & 673 & 47.5 \\
\hline Female & 744 & 52.5 \\
\hline \multicolumn{3}{|l|}{ Histological type } \\
\hline Adenocarcinoma & 943 & 66.5 \\
\hline Squamous cell carcinoma & 166 & 11.7 \\
\hline Urothelial carcinoma & 55 & 3.9 \\
\hline Melanoma & 54 & 3.8 \\
\hline Neuroendocrine tumor & 52 & 3.7 \\
\hline Tumor & 49 & 3.5 \\
\hline Sarcoma & 46 & 3.2 \\
\hline Germ cell tumor & 40 & 2.8 \\
\hline Mesothelioma & 12 & 0.9 \\
\hline \multicolumn{3}{|l|}{ Histologic grade } \\
\hline Well-moderately differentiated & 418 & 37.6 \\
\hline $\begin{array}{l}\text { Poorly differentiated/Undif- } \\
\text { ferentiated }\end{array}$ & 694 & 62.4 \\
\hline
\end{tabular}

BCH, Beijing Cancer Hospital; FUSCC, Fudan University Shanghai Cancer Center; $\mathrm{ZCH}$, Zhejiang Cancer Hospital

${ }^{a}$ The differentiation of 305 cases are not defined

\section{Analysis of discordant specimens}

A total of 79 tumor specimens had discordant predictions compared with reference diagnosis. Additional file 1: Table S1 investigated all cases with discordant results of the 90 -gene expression assay. The Top-5 common misclassified tumor types were gastroesophagus $(\mathrm{N}=10)$, head\&neck $(\mathrm{N}=8)$, liver $(\mathrm{N}=8)$, lung $(\mathrm{N}=7)$, and colorectum $(\mathrm{N}=7)$. Surprisingly, we noticed that eight head\&neck tumors were misclassified, among which seven cases were identified as gastroesophageal tumors. The histological types of misclassified specimens included poorly differentiated or undifferentiated $(\mathrm{N}=38)$, well-moderately differentiated tumors $(\mathrm{N}=19)$, and undefined $(\mathrm{N}=22)$.

\section{Discussion}

In the clinic, the identification of tumor type is crucial for optimal treatment selection when a patient diagnosed with a malignant tumor. The traditional diagnosis of tumor type requires a comprehensive analysis of the clinical and pathological findings. Imaging techniques including computed tomography (CT), magnetic resonance imaging (MRI), positron emission tomographycomputed tomography (PET-CT) scans are typically used for primary site detection in clinics. However, a recent meta-analysis of PET-CT in 1942 patients from 20 centers found a primary tumor detection rate of $40.9 \%$ (39.0\% to $42.9 \%$ ), which is still limited for identifying tumor tissue of origin [15].

In routine pathological diagnostic practice, morphological and IHC assessments were two relatively costefficient and no burden methods for patients, which could identify a tumor type in most cases. Nevertheless, the diagnosis of patients with poorly differentiated or undifferentiated tumors is not straightforward because tumors often lack the typical features [16]. Several studies reported an agreement of $69-71 \%$ in the characterization of poorly differentiated or undifferentiated carcinomas by performing the IHC and morphology analysis [17, 18].

In the recent decade, studies investigated that distinct tumor types have recognizable differences in gene expression patterns. When tumor metastasis occurs, the gene expression profile of the metastatic foci will maintain the gene expression profile of the primary tumor. Based on this finding, the tumor type of one tumor sample could be elucidated by comparing its gene expression pattern with the gene expression pattern in tumors with known tumor types $[19,20]$. Several gene expression assays such as the TOO and CancerTYPE ID have been developed based on mRNA and commercialized to predict the putative primary site for patients with uncertain diagnoses $[9,10]$. The TOO test reported by Monzon et al. was a microarray-based test on 1550 genes to differentiate 15 main tumor types. In a blinded validation study that included 547 frozen tumor specimens, the TOO test showed an $87.8 \%$ overall agreement with the reference diagnosis [9]. For the CancerTYPE ID assay, Erlander et al. developed a 92-gene real-time PCR assay for identifying the primary site of 28 common tumor types. A multisite validation study used the assay on 790 FFPE tumor specimens and demonstrated an overall sensitivity of $87 \%$ in primary site identification [10].

Recently, with the advance of NGS techniques, genomic alterations and DNA methylation have also been applied for tumor molecular classification. 

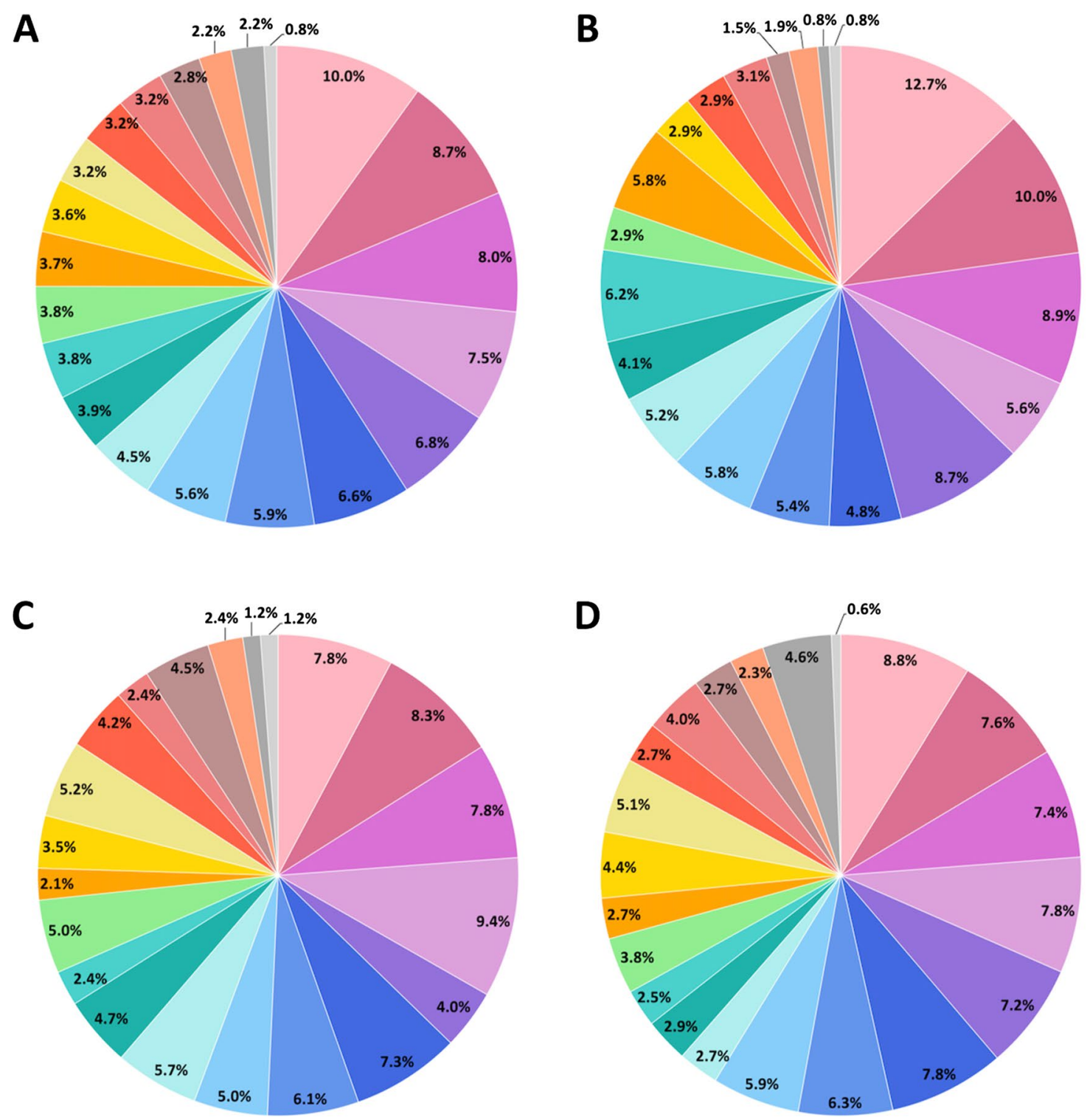
Lung
- Breast
- Colorectum
- Gastroesophagus
- Thyroid
- Cervix
- Liver
- Endometrium
Kidney
- Urinary
- Melanoma
- Ovary
- Neuroendocrine
- Prostate
Brain
- Sarcoma
- Pancreas
- Germ cell
Adrenal
Head Neck
Mesothelioma

Fig. 2 The distribution of tumor types in the A entire cohort, B Beijing Cancer Hospital, C Fudan University Shanghai Cancer Center, and D Zhejiang Cancer Hospital 
Table 2 Performance of the 90-gene expression assay in 21 tumor types

\begin{tabular}{|c|c|c|c|c|c|c|}
\hline Tumor types & Number & Agreement & Sensitivity & Specificity & PPV & NPV \\
\hline Adrenal & 31 & 31 & $100.0 \%$ & $100.0 \%$ & $100.0 \%$ & $100.0 \%$ \\
\hline Brain & 46 & 44 & $95.7 \%$ & $99.9 \%$ & $97.8 \%$ & $99.9 \%$ \\
\hline Breast & 123 & 121 & $98.4 \%$ & $99.8 \%$ & $98.4 \%$ & $99.8 \%$ \\
\hline Cervix & 93 & 89 & $95.7 \%$ & $99.0 \%$ & $87.3 \%$ & $99.7 \%$ \\
\hline Colorectum & 114 & 107 & $93.9 \%$ & $99.8 \%$ & $97.3 \%$ & $99.5 \%$ \\
\hline Endometrium & 79 & 77 & $97.5 \%$ & $99.6 \%$ & $93.9 \%$ & $99.9 \%$ \\
\hline Gastroesophagus & 106 & 96 & $90.6 \%$ & $99.0 \%$ & $88.1 \%$ & $99.2 \%$ \\
\hline Germ cell & 40 & 39 & $97.5 \%$ & $99.5 \%$ & $84.8 \%$ & $99.9 \%$ \\
\hline Head\&neck & 31 & 23 & $74.2 \%$ & $99.9 \%$ & $92.0 \%$ & $99.4 \%$ \\
\hline Kidney & 64 & 62 & $96.9 \%$ & $100.0 \%$ & $100.0 \%$ & $99.9 \%$ \\
\hline Liver & 84 & 76 & $90.5 \%$ & $99.8 \%$ & $97.4 \%$ & $99.4 \%$ \\
\hline Lung & 141 & 134 & $95.0 \%$ & $99.8 \%$ & $98.5 \%$ & $99.5 \%$ \\
\hline Melanoma & 54 & 48 & $88.9 \%$ & $100.0 \%$ & $100.0 \%$ & $99.6 \%$ \\
\hline Mesothelioma & 12 & 12 & $100.0 \%$ & $99.3 \%$ & $54.5 \%$ & $100.0 \%$ \\
\hline Neuroendocrine & 52 & 49 & $94.2 \%$ & $99.7 \%$ & $92.5 \%$ & $99.8 \%$ \\
\hline Ovary & 54 & 51 & $94.4 \%$ & $99.4 \%$ & $86.4 \%$ & $99.8 \%$ \\
\hline Pancreas & 45 & 40 & $88.9 \%$ & $99.9 \%$ & $97.6 \%$ & $99.6 \%$ \\
\hline Prostate & 51 & 51 & $100.0 \%$ & $99.9 \%$ & $98.1 \%$ & $100.0 \%$ \\
\hline Sarcoma & 46 & 43 & $93.5 \%$ & $99.8 \%$ & $93.5 \%$ & $99.8 \%$ \\
\hline Thyroid & 96 & 92 & $95.8 \%$ & $100.0 \%$ & $100.0 \%$ & $99.7 \%$ \\
\hline Urinary & 55 & 53 & $96.4 \%$ & $99.9 \%$ & $96.4 \%$ & $99.9 \%$ \\
\hline Total & 1417 & 1338 & Accuracy $=94.4 \%$ & & & \\
\hline
\end{tabular}

PPV, positive prediction value; NPV, negative prediction value

Alexander et al. applied machine learning to the assessment of genomic alteration data (468 cancer-associated genes) to predict the tissue of origin, with an overall accuracy of $74.1 \%$ in an independent cohort [6]. Sebastian et al. reported a DNA-methylation based test named "EPICUP" for identifying the tissue of origin of CUP. In a CUP validation cohort, EPICUP correctly predicted a primary site in $87 \%$ of CUP patients [21]. Moreover, researchers start to investigate the possibility of classifying tumors using less invasive procedures. One exciting approach was explored by M. C. et al., who analyzed the methylation patterns obtained from circulating cell-free DNA (cfDNA) to detect more than 50 cancer types [8]. In a validation cohort of 1354 cases, targeted methylation analysis demonstrated an overall sensitivity of $54.9 \%$ and a specificity of $>99 \%$.

This is, to our knowledge, the largest clinical validation study of a gene expression assay for tumor origin identification to date. Overall, the 90-gene expression assay correctly distinguishes tumor type in $94.4 \%$ of specimens, which is favorable with the other two commercially available tests (TOO and CancerTYPE ID) with $87 \%-87.8 \%$ accuracy $[9,10]$. Furthermore, the present study also established a large-scale prospective cohort $(\mathrm{N}=493)$ to assess the utilization of the 90-gene expression assay in a real clinical setting. Although the accuracy of the prospective cohort (92.1\%) was slightly lower than the retrospective cohort (95.7\%), it was still superior to the previous studies on tumor classification (87\%-87.8\%) [7]. Our results show that there is no significant difference in the performance of the gene expression assay for poorly differentiated/undifferentiated and wellmoderately differentiated tumors $(94.5 \%$ versus $95.5 \%$, respectively), suggesting that 90 -gene expression patterns of the tumor cells are robust and rarely affected by the loss of cell differentiation.

The present study still had several limitations. The first limitation was the exclusion of suboptimal specimens, such as biopsy samples (NCB or FNA), cytology samples, and samples with excess necrosis or few tumor contents. However, these types of samples are common and usually difficult to diagnosis in clinics. Further verification study is needed to validate the performance of the 90-gene expression assay for suboptimal specimens. 


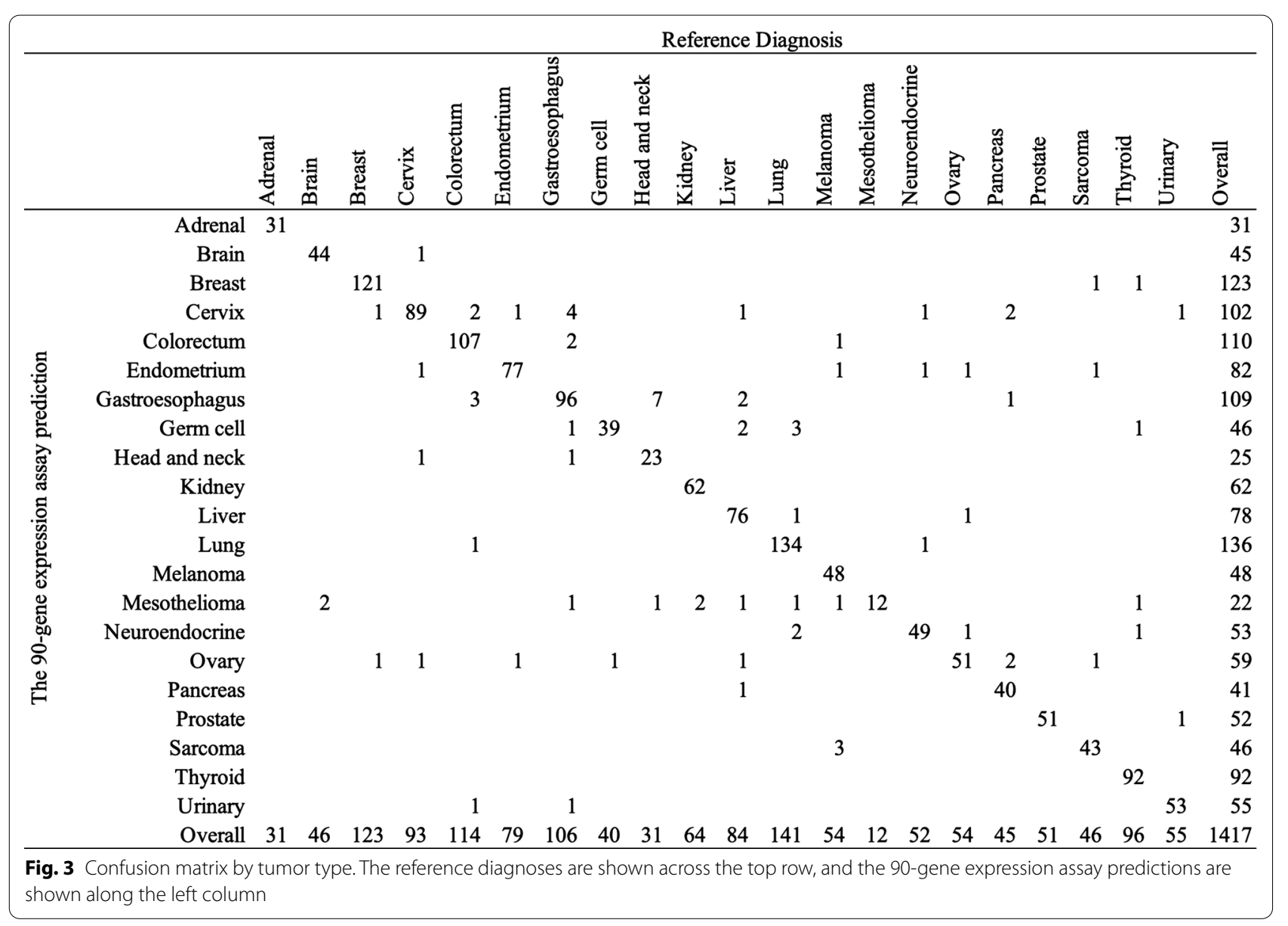

In addition, although the 90-gene expression assay achieved overall high classification accuracy cross different tumor types, we found that the performance in identifying the head\&neck tumor was not optimal. In this study, eight of 31 head\&neck tumors were misidentified, whereas seven of eight misclassified cases were identified as gastroesophageal tumors. Given the conjunction of esophagus and head\&neck in anatomy, the mRNA expression, DNA methylation, and somatic copy-number alterations data between esophagus squamous cell carcinoma and head\&neck squamous cell carcinoma were demonstrated with a strong resemblance [22]. Gene expression analyses with the 90-gene expression assay also reflect this biologic intersection and provide additional insight into the origin of these tumors. For this instance, additional effort was needed to improve the algorithm performance for distinguishing the head\&neck tumors and gastroesophageal tumors. Moreover, the predictions should be interpreted in conjunction with pathological diagnosis and clinical information when the tumor sample was predicted as head\&neck and/or gastroesophageal tumors during clinical use.

\section{Conclusion}

These findings showed robust performance of the 90-gene expression assay for identifying the tumor tissue of origin and support the use of molecular testing as an adjunct to tumor classification, especially to those poorly differentiated or undifferentiated tumors in clinical practice. 
Table 3 Performance of the 90-gene expression assay in clinicopathological subsets

\begin{tabular}{|c|c|c|c|}
\hline Clinical variables & Number & Agreement & Accuracy (\%) \\
\hline \multicolumn{4}{|l|}{ Study } \\
\hline Retrospective & 924 & 884 & 95.7 \\
\hline Prospective & 493 & 454 & 92.1 \\
\hline \multicolumn{4}{|l|}{ Histologic grade } \\
\hline Well-moderately differentiated & 418 & 399 & 95.5 \\
\hline Poorly differentiated/Undifferentiated & 694 & 656 & 94.5 \\
\hline \multicolumn{4}{|l|}{ Histological type } \\
\hline Adenocarcinoma & 943 & 898 & 95.2 \\
\hline Squamous cell carcinoma & 166 & 151 & 91.0 \\
\hline Urothelial carcinoma & 55 & 53 & 95.4 \\
\hline Melanoma & 54 & 48 & 88.9 \\
\hline Neuroendocrine tumor & 52 & 49 & 94.2 \\
\hline Tumor & 49 & 45 & 91.8 \\
\hline Sarcoma & 46 & 43 & 93.5 \\
\hline Germ cell tumor & 40 & 39 & 97.5 \\
\hline Mesothelioma & 12 & 12 & 100 \\
\hline \multicolumn{4}{|l|}{ Institution } \\
\hline $\mathrm{BCH}$ & 518 & 489 & 94.4 \\
\hline FUSCC & 424 & 403 & 95.0 \\
\hline $\mathrm{ZCH}$ & 475 & 446 & 93.9 \\
\hline
\end{tabular}

\section{Abbreviations}

BCH: Beijing Cancer Hospital; FUSCC: Fudan University Shanghai Cancer Center;; ZCH: Zhejiang Cancer Hospital.

\section{Supplementary Information}

The online version contains supplementary material available at https://doi. org/10.1186/s12967-022-03318-6.

Additional file 1: Table S1. Investigation of cases with discordant results of the 90 -gene expression assay.

\section{Acknowledgements}

Not applicable.

\section{Authors' contributions}

DML, WYS and XYZ designed the study. WS, WW, QFW, QY and YYL provided the specimens and collected clinical information. QF, YW, YS, QL, GZ and PQ performed the experiments. YFS, CHQ, WLR, ZZL, JYC, HYW and QHX analyzed all data. YFS and JYC wrote the initial manuscript draft. WS, WW, QFW, QHX, $X Y Z$, WYS and DML critically revised the manuscript and gave valuable insight into the study concept. All authors revised the manuscript. All authors read and approved the final manuscript.

\section{Funding}

This work was partially supported by research funding from Innovation Program of Shanghai Science and Technology Committee grants $20 Z 11900300$ (XY Z), Innovation Group Project of Shanghai Municipal Health Commission grants 2019CXJQ03 (XY Z), Shanghai Science and Technology Development Fund grants 19MC1911000 (XY Z, QF W, P Q) and Shanghai Municipal Key Clinical Specialty grants shslczdzk01301 (XY Z, QF W, P Q), the National Natural Science Foundation of China grants 81,401,963 (QF W) and 81,972,728 (QF W), Fudan University Shanghai Cancer Center grant YJYQ201603 (QF W), and Canhelp Genomics Co., Ltd.

\section{Declarations}

Ethics approval and consent to participate

In the study were obtained from the Clinical Research Ethics Committee of Beijing Cancer Hospital (BCH, Beijing, China), Fudan University Shanghai Cancer Center (FUSCC, Shanghai, China), and Cancer Hospital of the University of Chinese Academy of Sciences, Zhejiang Cancer Hospital (ZCH, Hangzhou, China)

\section{Consent for publication}

Not applicable.

\section{Competing interests}

Author YF S, CH Q, WL R, ZZ L, JY C, HY W and QH X were employed by the company Canhelp Genomics Co., Ltd. The remaining authors declare that the research was conducted in the absence of any commercial or financial relationships that could be construed as a potential conflict of interest.

\section{Author details}

${ }^{1}$ Department of Pathology, Key Laboratory of Carcinogenesis and Translational Research (Ministry of Education), Peking University Cancer Hospital \& Institute, No.52 Fucheng Road, Wu Ke Song, Haidian District, Beijing, China. ${ }^{2}$ Department of Pathology, Cancer Hospital of the University of Chinese Academy of Sciences (Zhejiang Cancer Hospital), No.1 East Road of Banshan, Hangzhou, Zhejiang, China. ${ }^{3}$ Department of Pathology, Fudan University Shanghai Cancer Center, No.270 Dong'An Road, Shanghai, China. ${ }^{4}$ Department of Oncology, Shanghai Medical College, Fudan University, Shanghai, China. Institute of Pathology, Fudan University, Shanghai, China. ${ }^{6}$ The Cancer of Unknown Primary Group of Pathology Committee, Chinese Research Hospital Association, Shanghai, China. ${ }^{7}$ The Canhelp Genomics Research Center, Canhelp Genomics Co., Ltd., Hangzhou, China. ${ }^{8}$ The Institute of Machine Learning and Systems Biology, College of Electronics and Information Engineering, Tongji University, Shanghai, China. ${ }^{9}$ Xuzhou Engineering Research Center of Medical Genetics and Transformation, Department of Genetics, Xuzhou Medical University, Xuzhou, China. 
Received: 21 January 2022 Accepted: 23 February 2022

Published online: 07 March 2022

\section{References}

1. Zhang S, Sun K, Zheng R, et al. Cancer incidence and mortality in China, 2015. J Natl Cancer Center. 2021;1(1):2-11. https://doi.org/10.1016/j.jncc 2020.12.001.

2. lizuka Y, lizuka H, Tsutsumi S, et al. Diagnosis of a previously unidentified primary site in patients with spinal metastasis: diagnostic usefulness of laboratory analysis, CT scanning and CT-guided biopsy. Eur Spine J. 2009;18(10):1431-5. https://doi.org/10.1007/s00586-009-1061-2.

3. El Rassy E, Pavlidis N. The current evidence for a biomarker-based approach in cancer of unknown primary. Cancer Treat Rev. 2018;67:21-8. https://doi.org/10.1016/j.ctrv.2018.04.011.

4. Selves J, Long-Mira E, Mathieu M-C, et al. Immunohistochemistry for diagnosis of metastatic carcinomas of unknown primary site. Cancers. 2018;10(4):108. https://doi.org/10.3390/cancers10040108.

5. Wafaie AW, Moussa KM, Ebeid EM, et al. Cancer of unknown primary origin: Can FDG PET/CT have a role in detecting the site of primary? Egypt J Radiol Nucl Med. 2018;49(1):190-5. https://doi.org/10.1016/j.jrnm.2017. 12.002.

6. Penson A, Camacho N, Zheng Y, et al. Development of genome-derived tumor type prediction to inform clinical cancer care. JAMA Oncol. 2020;6(1):84-91. https://doi.org/10.1001/jamaoncol.2019.3985.

7. Ye Q, Wang Q, Qi P, et al. Development and clinical validation of a 90-gene expression assay for identifying tumor tissue origin. J Mol Diagn. 2020;22(9):1139-50. https://doi.org/10.1016/j.jmoldx.2020.06.005.

8. Liu MC, Oxnard GR, Klein EA, et al. Sensitive and specific multi-cancer detection and localization using methylation signatures in cell-free DNA. Ann Oncol. 2020;31(6):745-59. https://doi.org/10.1016/j.annonc.2020.02. 011.

9. Monzon FA, Lyons-Weiler M, Buturovic $L$, et al. Multicenter validation of a 1,550-Gene expression profile for identification of tumor tissue of origin. Am Soc Clin Oncol Educ Book. 2009;27(15):2503-8. https://doi.org/10. 1200/JCO.2008.17.9762.

10. Kerr SE, Schnabel CA, Sullivan PS, et al. Multisite validation study to determine performance characteristics of a 92-gene molecular cancer classifier. Clin Cancer Res. 2012;18(14):3952-60. https://doi.org/10.1158/ 1078-0432.CCR-12-0920.

11. Wang $Q, X u M$, Sun $Y$, et al. Gene expression profiling for diagnosis of triple-negative breast cancer: a multicentre Retrospective Cohort Study. Front Oncol. 2019;9:115. https://doi.org/10.3389/fonc.2019.00354.

12. Zheng $Y$, Ding $Y$, Wang $Q$, et al. 90-gene signature assay for tissue origin diagnosis of brain metastases. J Transl Med. 2019;17(1):1-9. https://doi. org/10.1186/s12967-019-2082-1.

13. Qu N, Huang D, Xu Q, Wang J, et al. Gene expression profiling of cells of origin of squamous cell carcinomas in head-and-neck, esophagus, and lung. CCAB. 2020;9:89.

14. Zheng $Y$, Sun $Y$, Kuai $Y$, et al. Gene expression profiling for the diagnosis of multiple primary malignant tumors. Cancer Cell Int. 2021;21(1):1-9. https://doi.org/10.1186/s12935-021-01748-8.

15. Burglin SA, Hess S, Høilund-Carlsen PF, Gerke O. 18F-FDG PET/CT for detection of the primary tumor in adults with extracervical metastases from cancer of unknown primary: A systematic review and meta-analysis. Medicine. 2017;96(16): e6713. https://doi.org/10.1097/MD.00000000000 006713.

16. Rassy E, Pavlidis N. Progress in refining the clinical management of cancer of unknown primary in the molecular era. Nat Publ Group. 2020;17(9):541-54. https://doi.org/10.1038/s41571-020-0359-1.

17. Handorf CR, Kulkarni A, Grenert JP, et al. A multicenter study directly comparing the diagnostic accuracy of gene expression profiling and immunohistochemistry for primary site identification in metastatic tumors. Am J Surg Pathol. 2013;37(7):1067-75. https://doi.org/10.1097/ PAS.0b013e31828309c4

18. Weiss LM, Chu P, Schroeder BE, et al. Blinded comparator study of immunohistochemical analysis versus a 92-gene cancer classifier in the diagnosis of the primary site in metastatic tumors. J Mol Diagn. 2013;15(2):263-9. https://doi.org/10.1016/j.jmoldx.2012.10.001.
19. Ricketts CJ, De Cubas AA, Fan H, et al. The cancer genome atlas comprehensive molecular characterization of renal cell carcinoma. Cell Rep. 2018. https://doi.org/10.1016/j.celrep.2018.03.075.

20. Lee MS, Sanoff HK. Cancer of unknown primary. BMJ. 2020. https://doi. org/10.1136/bmj.m4050.

21. Moran S, Martinez-Cardús A, Sayols S, et al. Epigenetic profiling to classify cancer of unknown primary: a multicentre, retrospective analysis. Lancet Oncol. 2016;17(10):1386-95. https://doi.org/10.1016/S1470-2045(16) 30297-2.

22. Cancer Genome Atlas Research Network, Analysis Working Group: Asan University, BC Cancer Agency, et al. Integrated genomic characterization of oesophageal carcinoma. Nature. 2017;541(7636):169-175. doi:https:// doi.org/10.1038/nature20805.

\section{Publisher's Note}

Springer Nature remains neutral with regard to jurisdictional claims in published maps and institutional affiliations.

Ready to submit your research? Choose BMC and benefit from:

- fast, convenient online submission

- thorough peer review by experienced researchers in your field

- rapid publication on acceptance

- support for research data, including large and complex data types

- gold Open Access which fosters wider collaboration and increased citations

- maximum visibility for your research: over $100 \mathrm{M}$ website views per year

At BMC, research is always in progress.

Learn more biomedcentral.com/submissions 